\title{
AUTENTICAÇÃO DE ORÉGANO (Origanum vulgare L.) ORGÂNICO UTILIZANDO ESPECTROSCOPIA NIR E QUIMIOMETRIA
}

\author{
Ana Sophia Tovar Fernandez ${ }^{a}$, Andressa Rafaella da Silva Brunia, Vitória Maria Almeida Teodoro de Oliveira ${ }^{\text {a }}$, Paulo \\ Henrique Março e Patrícia Valderrama ${ }^{\mathrm{a}, *, 0}$ \\ a'Universidade Tecnológica Federal do Paraná, 87301-899 Campo Mourão - PR, Brasil
}

Recebido em 16/04/2020; aceito em 09/07/2020; publicado na web em 21/08/2020

\begin{abstract}
NIR SPECTROSCOPY AND CHEMOMETRICS IN THE ORGANIC ORIGANUM VULGARE L. In recent years, the consumption of organic foods has considerably increased. The basic difference between organic and non-organic food products is the way in which they are produced and processed, and the authenticity of these products is a current trend in food science and analytical chemistry. In this sense, the objective of this work was to propose an analytical methodology based on near-infrared spectroscopy (NIR) coupled with partial least squares with discriminant analysis (PLS-DA) for authentication of organic Origanum vulgare L., a spice known for its characteristic aroma and among the most popular condiments in the world, receiving prominence for its antimicrobial, antioxidant and anti-inflammatory properties. As a result, NIR/PLS-DA was able to promote a rapid and non-destructive analysis in the authentication of organic samples. Important spectral regions for this authentication were highlighted by VIP scores being from 1350 to $1430 \mathrm{~nm}$, and from 1450 to $1480 \mathrm{~nm}$ where it is observed absorption due to $\mathrm{ROH}, \mathrm{ArOH}, \mathrm{CONH}_{2}, \mathrm{RNH}_{2}, \mathrm{CONHR}, \mathrm{CH}$, $\mathrm{CH}_{2}$, and $\mathrm{CH}_{3}$.
\end{abstract}

Keywords: oregano; organic; authenticity; chemometrics; PLS-DA.

\section{INTRODUÇÃO}

Nos últimos anos, o consumo de alimentos orgânicos aumentou consideravelmente. A diferença entre produtos alimentícios orgânicos e não orgânicos é a forma como são produzidos e processados. ${ }^{1} \mathrm{Em}$ geral, os alimentos orgânicos são caracterizados por sua restrição ao uso de pesticidas e fertilizantes sintéticos em seu cultivo. Dessa forma, alimentos orgânicos não devem conter resíduos de fertilizantes sintéticos, pesticidas químicos, organismos geneticamente modificados, hormônios e antibióticos. ${ }^{2}$

Na agricultura, há uma preocupação crescente com o aumento do emprego de produtos químicos, quanto aos riscos à saúde humana e ao meio ambiente. Essa preocupação decorre de casos de doenças registradas em seres humanos e das alterações ambientais, que parecem ter como agentes etiológicos os agrotóxicos. ${ }^{3}$

Uma alternativa para a redução do uso de produtos químicos é a produção de alimentos orgânicos. No Brasil, de acordo com a Lei n ${ }^{\circ}$ 10.831, de 23 de dezembro de 2003, considera-se produto orgânico o alimento obtido em sistema orgânico de produção agropecuário, em que se adotam técnicas específicas, mediante a otimização do uso dos recursos naturais e socioeconômicos disponíveis e o respeito à integridade cultural das comunidades rurais, tendo por objetivo a sustentabilidade econômica e ecológica. ${ }^{4}$ Entre os alimentos orgânicos, o orégano é uma especiaria importante, pertencente à família Labiateae. O mesmo possui várias ramificações de seus galhos, sendo que suas folhas, comumente secas, são utilizadas como tempero. Ele está entre os condimentos mais populares do mundo, conhecido pelo seu aroma característico, proveniente do seu significativo conteúdo de carvacrol. ${ }^{5}$ No Brasil, o orégano está entre as plantas condimentares mais utilizadas, em especial para aprimorar o sabor de massas e carnes. ${ }^{6}$

Praticamente todo o orégano comercializado no país é importado. Entretanto, a espécie Origanum vulgare L. é cultivada no Brasil principalmente nas regiões sul e sudeste, e apresenta algumas exigências

*e-mail: pativalderrama@gmail.com climáticas, produzindo melhor qualidade da planta em invernos secos e ensolarados. ${ }^{7}$

Em relação a esse condimento, são inúmeros os estudos que o utiliza na medicina, para o tratamento de tosses, desordens do trato digestivo e problemas menstruais. Além disso, é apresentado diversas vezes na literatura por possuir inúmeros compostos benéficos a saúde de quem o consome, como o carvacrol e timol que possuem atividades anti-inflamatórias, antibacterianas e antioxidantes, além dos efeitos fungicidas e anti-câncer que lhe é presente..$^{8-12}$

Um estudo mostrou que ambientes com luz modificada e adubação orgânica contribuíram para a redução de trocas gasosas e favoreceram o crescimento do orégano. Nessas condições, as plantas apresentaram maior número de folhas, e maior produção de massa seca. ${ }^{6}$

Considerando a produção de alimentos orgânicos, até o ano de 2017 o mundo apresentava uma área agricultável mundial destinada a cultivos orgânicos de 69,8 milhões de hectares. Um levantamento aponta a evolução das áreas destinadas à produção orgânica, entre 2007 e 2017, sendo que dos vinte países com as maiores extensões de área em 2017, o Brasil encontra-se na décima segunda posição. Entretanto, apesar de se verificar um crescimento expressivo de áreas agrícolas e produtores dedicados à agricultura orgânica, a expansão depende do enfrentamento de desafios, dentre eles a padronização dos critérios de certificação. ${ }^{13}$

Dessa forma, a autenticidade dos alimentos orgânicos passa a ser uma preocupação atual, e assim o objetivo desse trabalho é propor uma metodologia analítica baseada na espectroscopia na região do infravermelho próximo (NIR) empregando a ferramenta quimiométrica de mínimos quadrados parciais com análise discriminante (PLS-DA) para autenticação de orégano orgânico.

A espectroscopia NIR vem sendo aplicada na autenticação de alimentos, ${ }^{14-18}$ apresentando inúmeras vantagens, como por exemplo, ser uma técnica rápida, simples, que permite múltiplas respostas simultaneamente, com pouca ou nenhuma preparação da amostra. Em conjunto com a espectroscopia NIR, normalmente, utiliza-se de ferramentas da quimiometria para melhorar a extração de informações relevantes e úteis dos dados espectrais medidos. 
Na presente proposta, considerando que o objetivo é propor uma metodologia para autenticação, a ferramenta PLS-DA torna-se apropriada por fornecer uma avaliação supervisionada quando os dados se ajustam a um modelo linear, sendo capaz de identificar amostras anômalas (outliers), e mostrar resultados eficientes na modelagem quando tem-se grande variabilidade dentro de uma mesma classe de amostras ${ }^{19}$ (por exemplo, amostras da classe orgânica podem ser oriundas de diferentes regiões, e mesmo promovendo uma separação entre si de acordo com a região, ainda podem ser modeladas como uma única classe com a ferramenta PLS-DA). Ainda, critérios para a escolha apropriada do número de variáveis latentes e os parâmetros de validação do modelo são características bem estabelecidas que agregam vantagem à ferramenta.

\section{PARTE EXPERIMENTAL}

\section{Amostras}

Para a realização desse estudo foram utilizadas 140 amostras comerciais de orégano de marcas e lotes diferentes oriundas do Canadá, sendo 50 amostras orgânicas e 90 não orgânicas. Maiores informações sobre o sistema de produção orgânico no Canadá podem ser obtidas em https://www.inspection.gc.ca/organic-products/standards/eng/1 $300368619837 / 1300368673172$.

As amostras foram submetidas à trituração utilizando um liquidificador industrial e, posteriormente, passaram por uma peneira padronizada em 60 mesh, para assegurar homogeneidade dos espectros.

\section{Coleta dos espectros}

Todas as medidas foram realizadas no mesmo dia em ambiente climatizado a $22^{\circ} \mathrm{C}$. Os espectros foram medidos com o equipamento microNIR JDSU no modo reflectância na região de 1100 a $1660 \mathrm{~nm}$ (32 scans por amostra e resolução de $4 \mathrm{~nm}$ ), obtendo um total de 140 espectros.

\section{Processamento dos dados}

Os espectros coletados foram processados com o auxílio do software MATLAB R2007B empregando a ferramenta quimiométrica PLS-DA através do PLS-Toolbox 5.2. Esses espectros adquiridos no modo refletância foram convertidos em absorbância, e posteriormente pré-processados através de suavização com o algoritmo savgol (polinômio de primeira ordem e intervalo de 11 pontos) e primeira derivada. ${ }^{20}$

As amostras foram divididas em conjuntos de modelagem e validação através do algoritmo de Kennard-Stone. ${ }^{21}$ Esse algoritmo seleciona a primeira amostra de maior distância em relação ao centro dos dados, a próxima amostra será novamente aquela de maior distância do último ponto e assim sucessivamente, até completar o número de amostras do conjunto de modelagem.

A qualidade do modelo foi avaliada através da sensibilidade e especificidade. A sensibilidade se refere ao número de amostras previstas como sendo da classe, dividido pelo número de amostras que realmente pertencem à classe, já a especificidade do modelo se refere ao número de amostras previstas como não sendo da classe, dividido pelo número real de amostras que não são da classe. ${ }^{22}$

O modelo foi construído com os dados centrados na média e para a escolha do número de variáveis latentes (VLs) utilizou-se o critério de decisão Bayesiana. ${ }^{23}$

\section{PLS-DA}

O método de mínimos quadrados parciais com análise discriminante (PLS-DA) é um método quimiométrico de reconhecimento de padrão supervisionado, ${ }^{24}$ que utiliza as informações prévias das amostras na decomposição dos dados em scores e loadings. ${ }^{25}$ Ele é baseado no método de regressão de Mínimos Quadratos Parciais $(\mathrm{PLS})^{26}$ e ambos são fundamentados no método de Análise de Componentes Principais (PCA), um sistema não supervisionado de reconhecimentos de padrões. ${ }^{27}$

PLS-DA pode ser aplicado a dados de primeira ordem, como os espectros NIR, nos quais um vetor de respostas é obtido para cada amostra. ${ }^{28}$ Para sua utilização os vetores de dados (cada um dos espectros NIR) são organizados no formato de uma matriz $(\mathbf{X})$, em que cada linha dessa matriz representa as amostras e cada coluna corresponde à absorbância nos diferentes comprimentos de onda.

No PLS-DA, na matriz $\mathbf{Y}$ estão as informações relacionadas a classe de cada amostra. Assim, essa matriz assume valores de 'zero' e 'um', e esses valores indicam se a amostra pertence ou não a classe. Um valor limite (threshold) é calculado entre os valores previstos e os valores acima desse limite indicam que a amostra pertence à classe modelada, enquanto que valores abaixo correspondem às amostras que não pertencem à classe modelada. ${ }^{23}$ A Figura 1 exemplifica a organização das matrizes $\mathbf{X}$ e $\mathbf{Y}$ para a execução do PLS-DA.

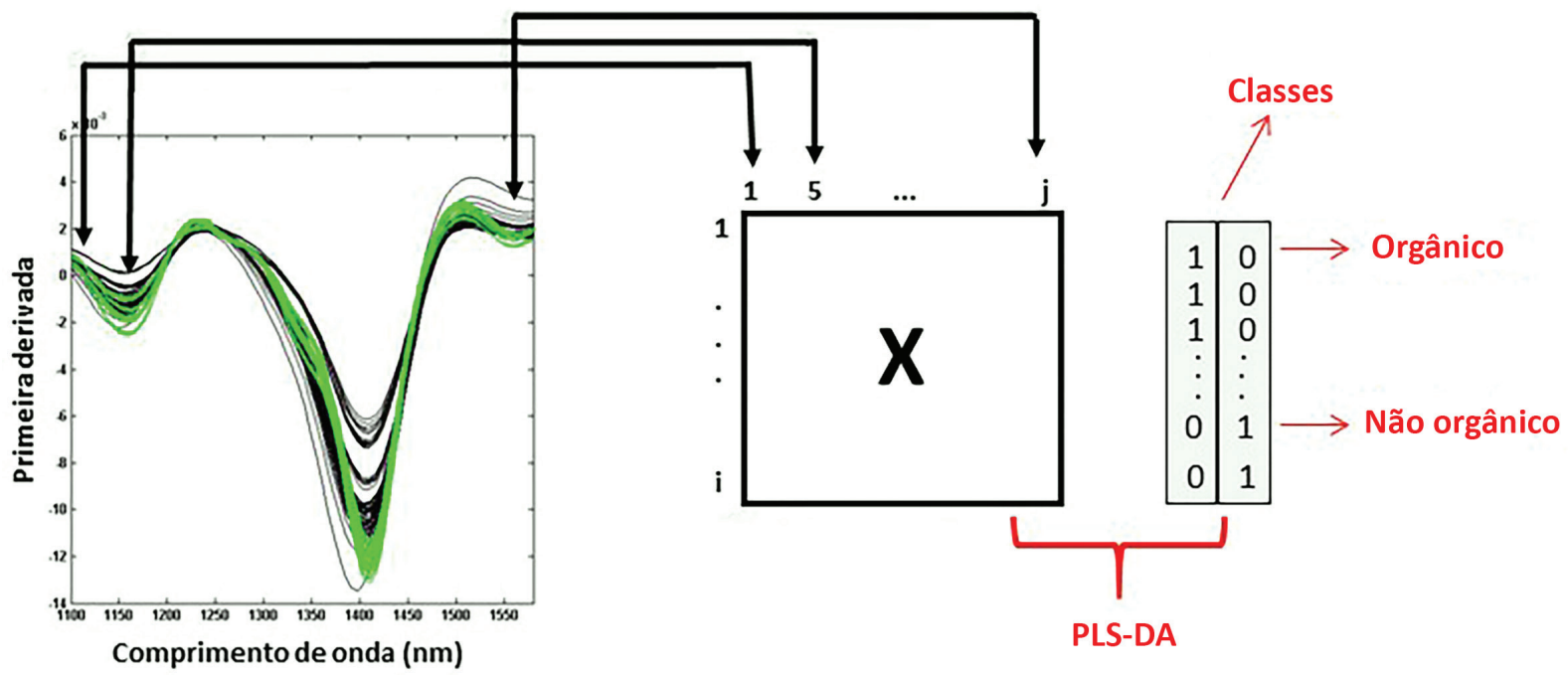

Figura 1. Organização das matrizes $\boldsymbol{X}$ e $\boldsymbol{Y}$ para execução do PLS-DA 


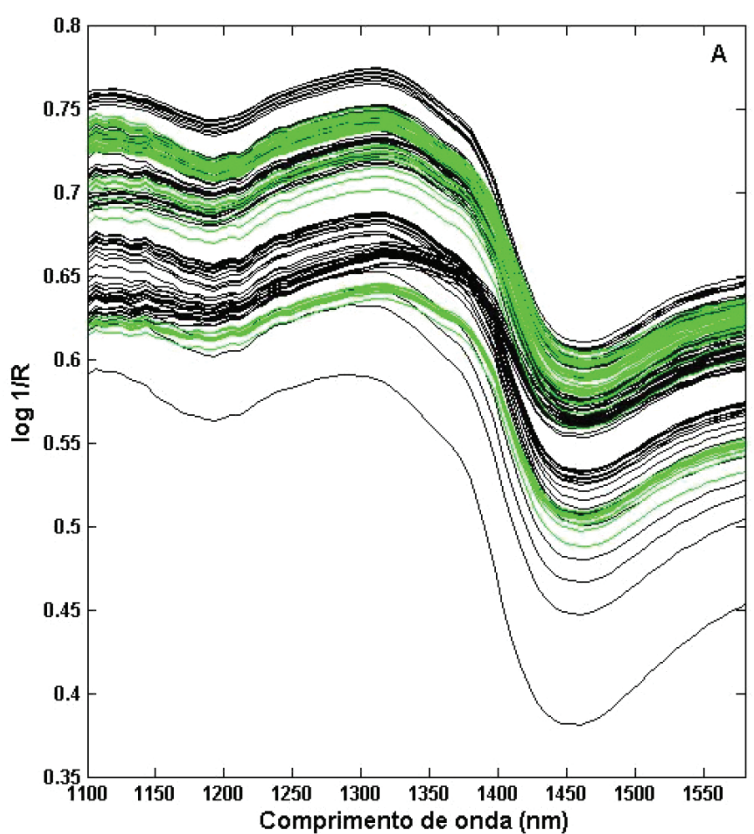

Figura 2. Espectros NIR das amostras de orégano. (A) Espectros brutos. amostras não-orgânicas

\section{RESULTADOS E DISCUSSÃO}

A Figura 2 mostra os espectros NIR na região de 1100 a $1580 \mathrm{~nm}$ das amostras de orégano, após pré-processamento. Pequenas diferenças podem sem observadas ao longo dos espectros. Entretanto, não se destaca uma região espectral que sugira a identificação das amostras orgânicas apenas visualmente. Assim, a falta de seletividade na espectroscopia NIR e seu alto grau de similaridade entre os sinais das amostras, dificultam a interpretação espectral ${ }^{29} \mathrm{e}$, portanto, uma ferramenta quimiométrica como o PLS-DA pode contribuir para uma maior segurança na avaliação dos resultados. ${ }^{21}$

O modelo PLS-DA foi construído com os dados centrados na média, empregando apenas duas VLs e também validação cruzada em blocos contínuos de 10 amostras. A avaliação de outliers foi realizada a partir do gráfico de Q Residuals versus Hotelling $\mathrm{T}^{\wedge} 2$, podendo ser visualizada na Figura 3. Através dessa figura, verifica-se que duas amostras não-orgânicas da etapa de modelagem apresentam resultados acima do limite de $95 \%$ para Q Residuals. Entretanto, essas mesmas amostras apresentam resultados dentro do limite de $95 \%$ para Hotelling $\mathrm{T}^{\wedge} 2$. Dessa forma, nenhuma amostra foi identificada como outlier, uma vez que para tal atribuição a amostra deve apresentar, simultaneamente, resultados acima do limite de $95 \%$ para ambos os testes, Q Residuals e Hotelling $\mathrm{T}^{\wedge} 2$.

A Figura 4 ilustra a distribuição das amostras de modelagem e validação para o modelo de autenticação, em que se pode observar uma separação nítida entre as amostras das classes orgânicas das não-orgânicas. Ainda, outros estudos abordam sobre a possibilidade do emprego da espectroscopia NIR na autenticação de alimentos orgânicos, como por exemplo açúcares,${ }^{30}$ morangos,${ }^{31}$ maçãs,${ }^{32}$ aspargos,${ }^{33}$ e leite. ${ }^{34}$

No modelo construído a sensibilidade e especificidade foram iguais a 1 para ambas as classes orgânicas e não-orgânicas, isso significa que o modelo foi eficaz para classificar corretamente todas as amostras das duas classes.

Resultados de sensibilidade e especificidade iguais a 1 foram alcançados na autenticação de açúcares (cristal, demerara e mascavo) orgânicos. ${ }^{30}$ Valores próximos a 1 também foram ratificados na autenticação de morangos orgânicos, ${ }^{31}$ e na autenticação de óleo de soja de origem transgênica e não transgênicas. ${ }^{19}$

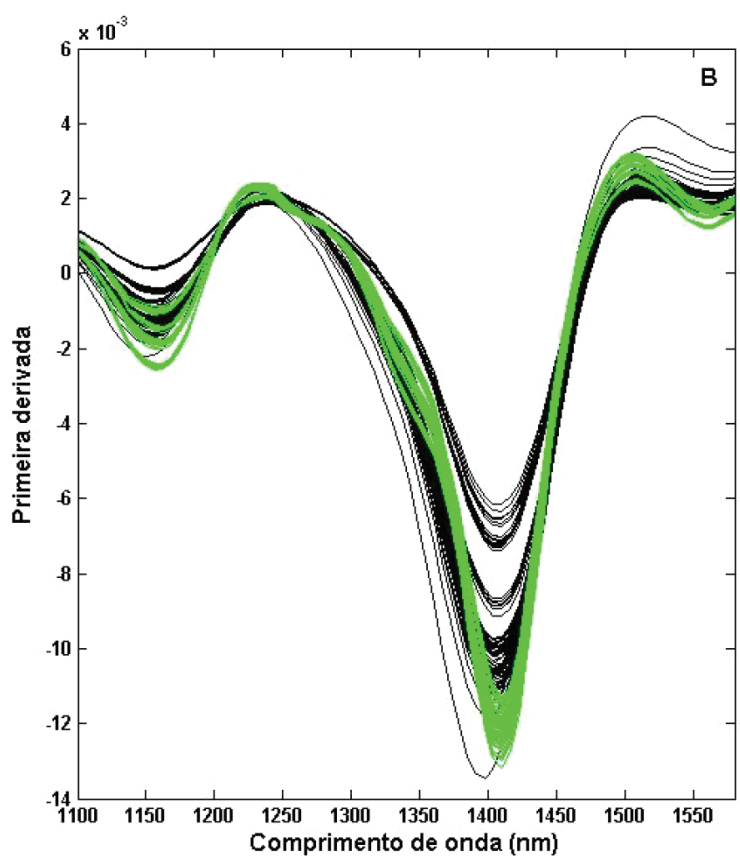

(B) Espectros após primeira derivada e suavização. (-) amostras orgânicas. (一)

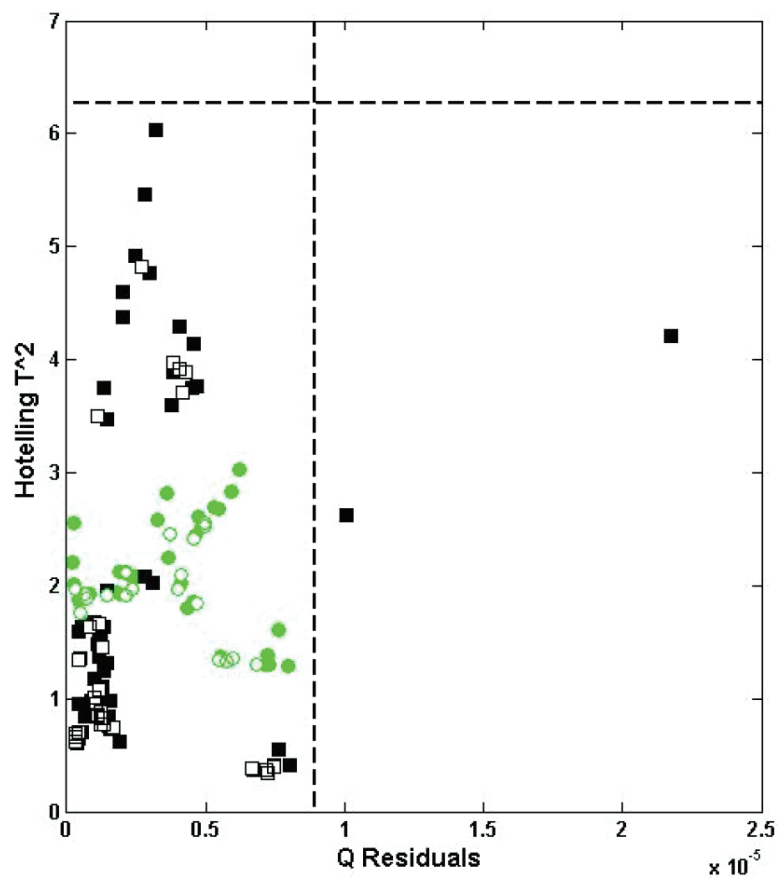

Figura 3. Q Residuals versus Hotelling $T^{\wedge 2}$. (○) amostras orgânicas na modelagem. $(\bigcirc)$ amostras orgânicas na validação. ( $\mathbf{\square})$ amostras não-orgânicas na modelagem. ( $\square$ ) amostras não-orgânicas na validação. (---) threshold

Filés de robalo europeu orgânicos e liofilizados tiveram sua autenticidade avaliada através da espectroscopia NIR e modelagem por SIMCA (Soft Independent Modeling of Class Analogy). Essa metodologia classificou corretamente apenas $65 \%$ das amostras. ${ }^{35}$ Uma metodologia baseada na composição em ácidos graxos e PLSDA foi proposta para autenticação de leite de vacas que receberam alimentação orgânica na Holanda. O modelo foi capaz de classificar corretamente apenas $70 \%$ das amostras orgânicas. ${ }^{36}$

A importância da variável na projeção (VIP - Variable Importance in Projection) foi determinada, ${ }^{37}$ e destaca as regiões espectrais (Figura 5) importantes para o modelo NIR/PLS-DA na autenticação 

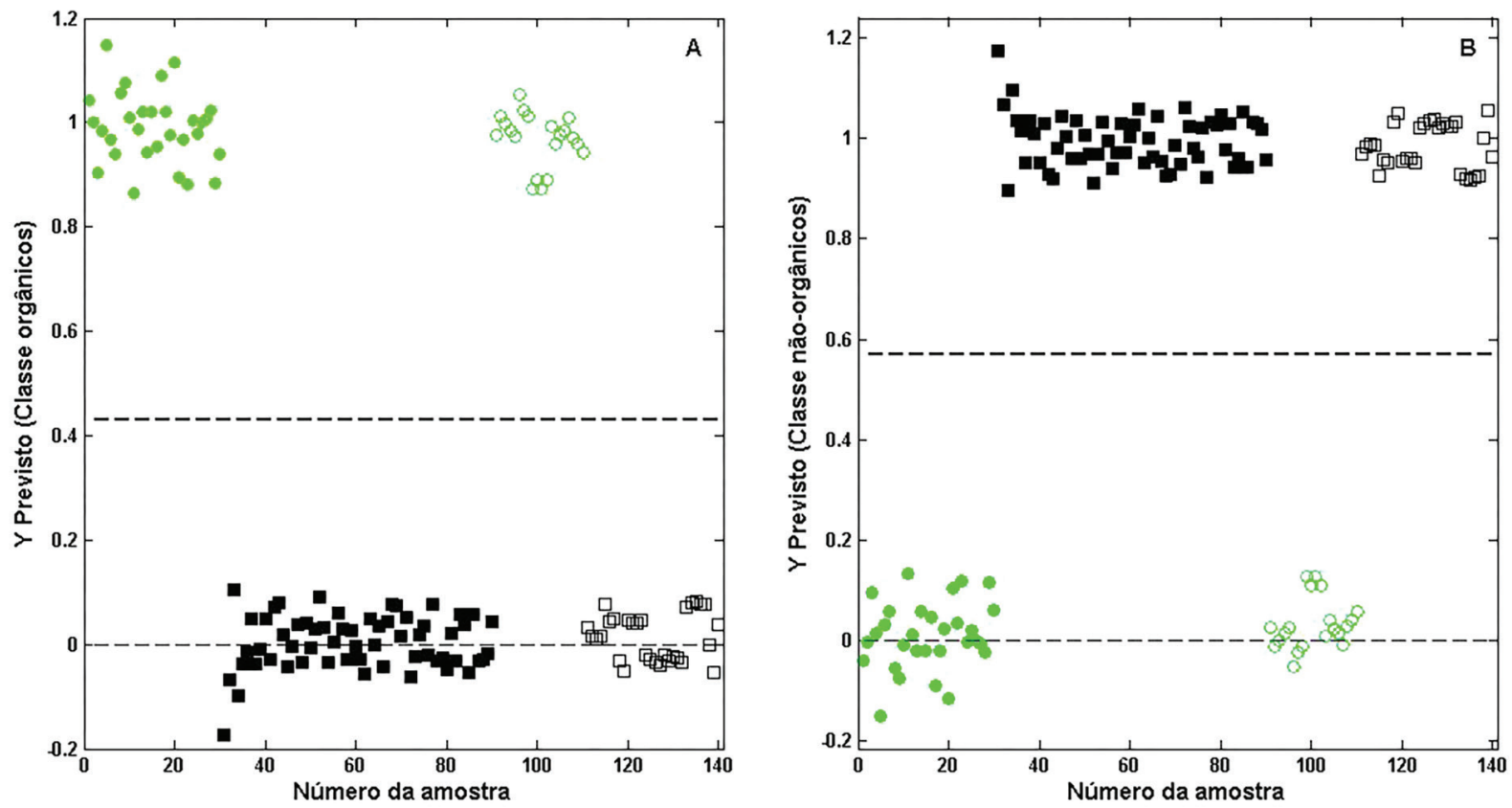

Figura 4. Distribuição das amostras de modelagem e validação no modelo PLS-DA. (A) amostras orgânicas. (B) amostras não orgânicas. (O) amostras orgânicas na modelagem. (○) amostras orgânicas na validação. ( $\mathbf{\square})$ amostras não-orgânicas na modelagem. ( $\square)$ amostras não-orgânicas na validação. (---) threshold

do orégano orgânico. De acordo com esse gráfico, as variáveis que apresentam valores de VIP maiores que ' 1 ' são as mais importantes na modelagem. Dessa forma, a região do segundo overtone entre 1350 - $1430 \mathrm{~nm}$ e 1450 - $1480 \mathrm{~nm}$ destacam-se como importantes na autenticação do orégano orgânico e referem-se a absorção de $\mathrm{ROH}$, $\mathrm{ArOH}, \mathrm{CONH}_{2}, \mathrm{RNH}_{2}, \mathrm{CONHR}, \mathrm{CH}, \mathrm{CH}_{2}, \mathrm{e} \mathrm{CH}_{3} \cdot{ }^{38}$

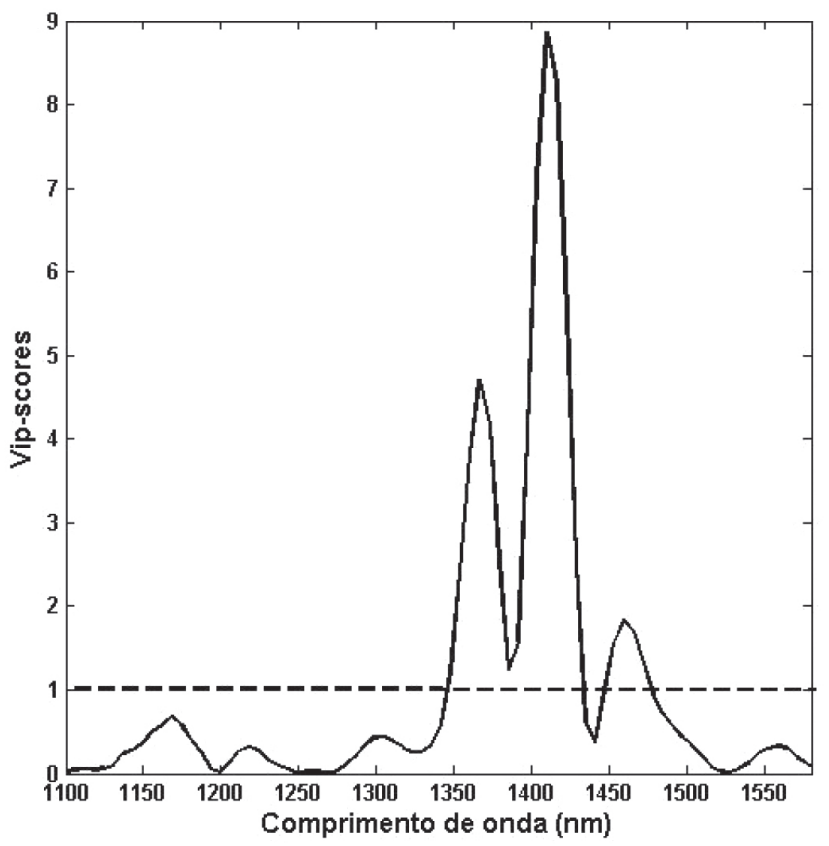

Figura 5. VIP scores

\section{CONCLUSÃO}

A espectroscopia NIR associada com a ferramenta quimiométrica PLS-DA mostrou-se capaz de autenticar amostras de orégano orgânico. O método proposto permitiu classificar corretamente $100 \%$ das amostras orgânicas em uma análise rápida e não destrutiva, com um mínimo de preparo da amostra. Através do VIP scores concluiu-se que a região espectral do segundo overtone foi a responsável pela autenticação das amostras orgânicas.

\section{AGRADECIMENTOS}

O presente trabalho foi realizado com apoio da Coordenação de Aperfeiçoamento de Pessoal de Nível Superior - Brasil (CAPES) - Código de Financiamento 001 (bolsa de mestrado de Andressa Rafaella da Silva Bruni) e edital PAEC OEA-GCUB (bolsa de mestrado de Ana Sophia Tovar Fernandez). Os autores agradecem à UTFPR pela bolsa de estudos da mestranda Vitória Maria Almeida Teodoro de Oliveira. Patrícia Valderrama agradece à Fundação Araucária (processo 033/2019).

\section{REFERÊNCIAS}

1. Popa, M. E.; Mitelut, A. C.; Popa, E. E.; Stan, A.; Popa, V. I.; Trends Food Sci. Technol. 2019, 84, 15.

2. González, N.; Marquès, M.; Nadal, M.; Domingo, J. L.; Food Chem. Toxicol. 2019, 125, 370.

3. Borguini, R. G.; Torres, E. A. F. da S.; Segurança Alimentar e Nutricional 2015, 13, 64.

4. Brasil Lei $n^{\circ} 10.831,23$ de dezembro de 2003. Dispõe sobre agricultura orgânica e dá outras providências. Presidência da República, Casa Civil, Subchefia para Assuntos Jurídicos, 2003, 8 .

5. Martins, E. R.; Castro, D. M.; Castellani, D. C.; Dias, J. E.; Plantas Medicinais, Editora da UFV: Viçosa, 1998.

6. Oliveira, V. C.; Santos, A. R.; Souza, G. S.; Santos, R. M.; Revista Colombiana de Ciencias Hortícolas 2017, 11, 400.

7. http://www.infobibos.com/Artigos/2009_2/Oregano/Index.htm, acessada em agosto 2020.

8. Verma, R. S.; Rahman, L.; Verma, R. K.; Chanotiya, C. S.; Chauhan, A.; Yadav, A.; Yadav, A. K.; Singh, A.; Curr. Sci. 2010, 98, 2010.

9. Han, X.; Parker, T. L.; Biochim. Open 2017, 4, 73.

10. Olmedo, R.; Nepote, V.; Grosso, N. R.; Food Chem. 2014, 156, 212.

11. Govaris, A.; Solomakos, N.; Pexara, A.; Chatzopoulou, P. S.; Int. J. Food Microbiol. 2010, 137, 175.

12. Pezzani, R.; Vitalini, S.; Iriti, M.; Phytochem. Rev. 2017, 16, 1253. 
13. https://www.ipea.gov.br/portal/images/stories/PDFs/TDs/td_2538.pdf, acessada em agosto 2020.

14. Firmani, P.; Bucci, R.; Marini, F.; Biancolillo, A.; J. Food Compos. Anal. 2019, 82, 103235 .

15. Firmani, P.; La Piscopia, G.; Bucci, R.; Marini, F.; Biancolillo, A.; Microchem. J. 2020, 152, 104339.

16. Srata, L.; Farres, S.; Fethi, F.; Vib. Spectrosc. 2019, 100, 99.

17. Maione, C.; De Paula, E. S.; Gallimberti, M.; Batista, B. L.; Campiglia, A. D.; Barbosa, F.; Barbosa, R. M.; Expert Systems With Applications 2016, 49,60 .

18. Firmani, P.; De Luca, S.; Bucci, R.; Marini, F.; Biancolillo, A.; Food Control 2019, 100, 292.

19. Alves, F. C. G. B. S.; Valderrama, P.; Anal. Methods 2015, 7, 9702.

20. Savitzky, A.; Golay, M. J. E.; Anal. Chem. 1964, 36, 1627.

21. Kennard, R. W.; Stone, L. A.; Technometrics 1969, 11, 137.

22. Almeida, M. R.; Fidelis, C. H. V.; Barata, L. E. S.; Poppi, R. J.; Talanta 2013, 117, 305.

23. Ferreira MMC. Quimiometria - Conceitos, métodos e aplicações, Editora Unicamp: Campinas, 2015.

24. Barker, M.; Rayens, W.; J. Chemom. 2003, 17, 166.

25. Valderrama, L.; Valderrama, P.; Chemom. Intell. Lab. Syst. 2016, 156, 188

26. Geladi, P.; Bruce, K.; Anal. Chim. Acta 1986, 186, 1

27. Wold, S.; Esbensen, K. I. M.; Geladi, P.; Chemom. Intell. Lab. Syst. 1987, 2, 37 .
28. Valderrama, L.; Gonçalves, R. P.; Março, P. H.; Valderrama, P.; Rebrapa 2014, 5,32 .

29. Farres, S.; Srata, L.; Fethi, F.; Kadaoui, A.; Vib. Spectrosc. 2019, 102, 79.

30. De Oliveira, V. M. A. T.; Baqueta, M. R.; Março, P. H.; Valderrama, P.; Anal. Methods 2020, 12, 701 .

31. Amodio, M. L.; Ceglie, F.; Chaudhry, M. M. A.; Piazzolla, F.; Colelli, G.; Postharvest Biol. Technol. 2017, 125, 112.

32. Song, W.; Wang, H.; Maguire, P.; Nibouche, O.; Proc. IEEE Sensors 2017, 31.

33. Sánchez, M. T.; Garrido-Varo, A.; Guerrero, J. E.; Pérez-Marín, D.; Postharvest Biol. Technol. 2013, 85, 116.

34. Liu, N.; Parra, H. A.; Pustjens, A.; Hettinga, K.; Mongondry, P.; van Ruth, S. M.; Talanta 2018, 184, 128

35. Trocino, A.; Xiccato, G.; Majolini, D.; Tazzoli, M.; Bertotto, D.; Pascoli, F.; Palazzi, R.; Food Chem. 2012, 131, 427.

36. Capuano, E.; Van Der Veer, G.; Boerrigter-Eenling, R.; Elgersma, A.; Rademaker, J.; Sterian, A.; Van Ruth, S. M.; Food Chem. 2014, 164, 234.

37. Vitale, R.; Bevilacqua, M.; Bucci, R.; Magrì, A. D.; Magrì, A. L.; Marini, F.; Chemom. Intell. Lab. Syst. 2013, 121, 90.

38. https://www.metrohm.com/en/documents/81085026, acessada em agosto 2020. 